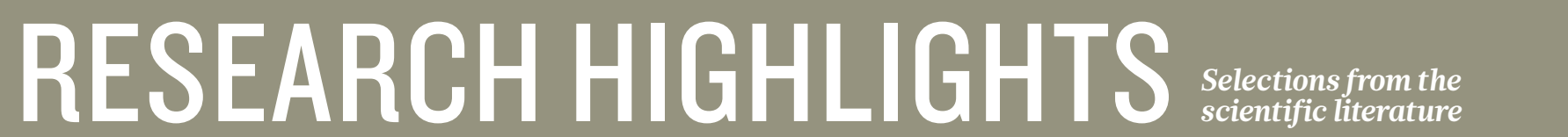

ECOTOXICOLOGY

\section{Venom pesticide safe for bees}

A pesticide based on toxins from spider venom seems to be safe for honeybees, an important crop pollinator. The pesticide could potentially replace other insecticides that have been banned recently because of their harmful effects on bees.

Angharad Gatehouse at Newcastle University, UK, and her colleagues fed or injected honeybees (Apis mellifera) with a biopesticide consisting of a spider toxin linked to a carrier protein. The researchers found that the chemical had little or no effect on mortality, learning and memory.

In larvae, the toxin was broken down in the gut, whereas in adults, it made its way to the brain. The lack of effects is probably because the compound does not block calcium channels in bee brain cells as it does in other insects, the authors say.

Proc. R. Soc. B 281, 20140619 (2014)

\section{PLANETARY SCIENCE}

\section{Early planet helped make Moon}

Small chemical differences between Earth and the Moon support the idea that the Moon formed from remnants of a large early planet, or protoplanet, that smashed into Earth 4.5 billion years ago.

Previous studies did not find differences in isotopic chemistry between Earth

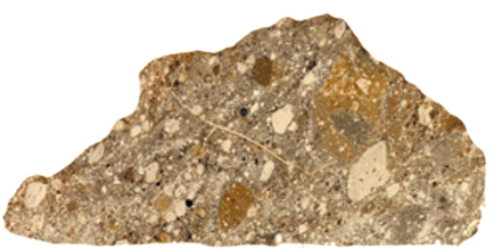

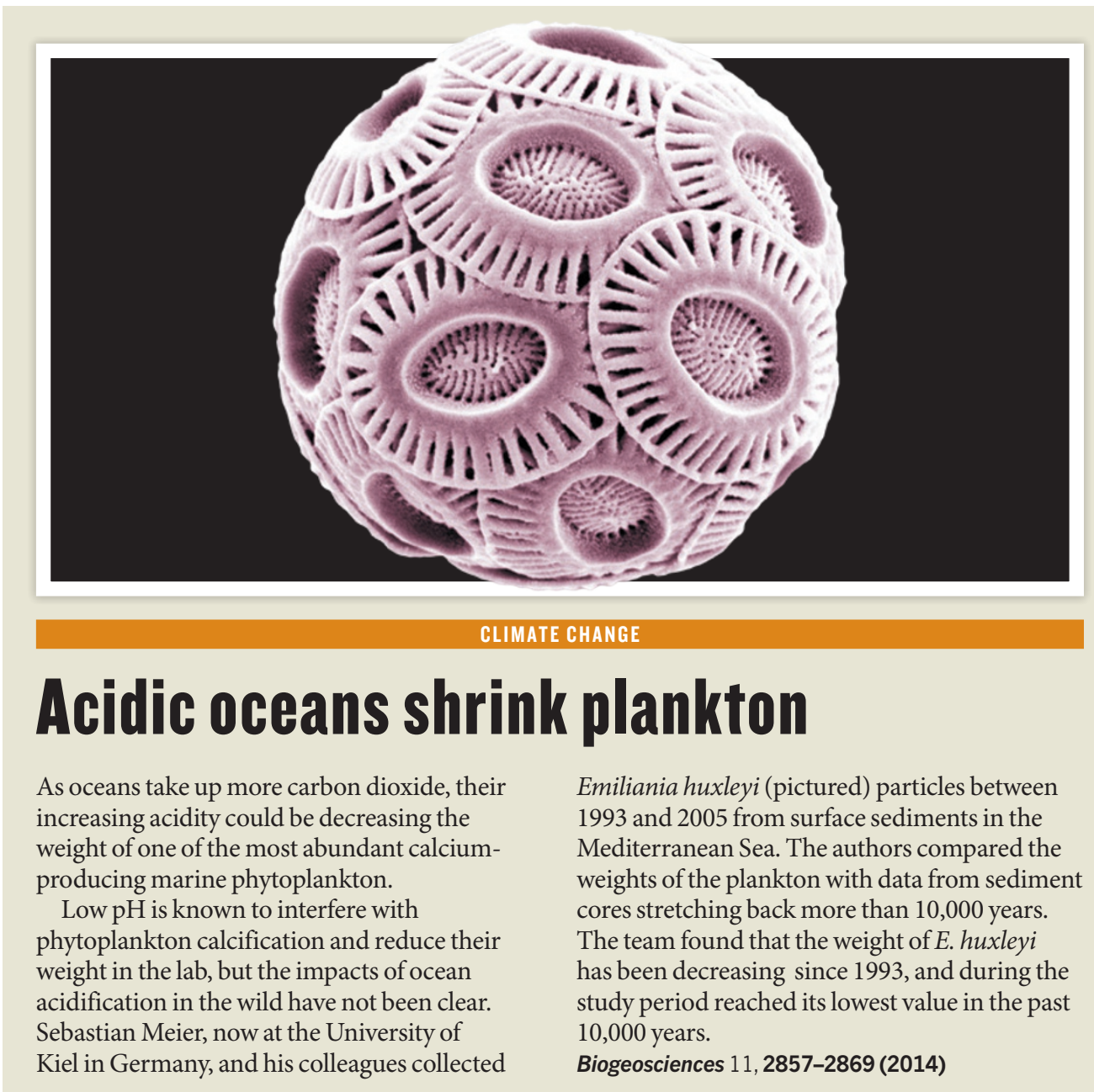

and Moon rocks, puzzling many who think that the protoplanet must have left a chemical mark on the Moon. Daniel Herwartz at the University of Cologne in Germany and his team measured levels of the isotope oxygen-17 in a range of Earth rocks, lunar meteorites that fell to Earth (pictured) and three lunar samples collected by astronauts. The authors found 12 parts per million more oxygen-17 in the Moon rocks than in Earth samples.

The impacting protoplanet, called Theia, could have been chemically similar enough to Earth, at least in terms of oxygen, that any imprint from Theia in the Moon's chemistry would have been difficult to detect, the authors say.

Science 344, 1146-1150 (2014)

VIROLOGY

\section{Chemicals wake up dormant HIV}

Compounds that boost the 'noise' in genetic activity of HIV can reactivate the latent virus. Such drugs could make it easier to stamp out quiescent HIV infections.

In patients, HIV can hide from antiretroviral drugs in a latent state for years. Changes in HIV gene expression can, when the expression reaches a certain threshold, lead to the activation of dormant HIV, making the virus a target for drugs. So, Leor Weinberger from the University of California in San Francisco and his team screened a library of small molecules and found more than 80 that modulate HIV gene-expression fluctuations. These "noise enhancers", in combination with existing reactivation compounds, reawakened latent HIV-infected cells better than the existing drugs alone, and were less toxic to uninfected cells.

Chemicals that modulate this genetic noise could be used in combination with other antiviral drugs to reawaken and kill HIV and other viruses that enter a latent state. Science http://doi.org/s4k (2014) 诺 